\title{
GW23-e2390 SIGNIFICANCE OF BLOOD BRAIN NATRIURETIC PEPTIDE IN DIAGNOSIS OF ACUTE DYSPNOEA
}

doi:10.1136/heartjnl-2012-302920o.6

Zhicheng Wu, Donyang Xie, Zixiang zhang. The First Affiliated Hospital of Gannan Meidical University

Objectives To explore clinical significance of differential diagnosis of plasma BNP concentration change between patients with cardiac dyspea and lung-derived dyspea.

Methods Select Thoracic and Cardiovascular Surgery, Respiratory Medicine, Cardiology, Emergency Department in The first affiliated Hospital of Gannan Meidical University with acute respiratory distress between January 2011 to January 2012, To rule out trauma, cardiac tamponade, acute coronary syndrome, congenital heart disease, renal insufficiency diseases, were male and 126 cases of the male, 105 cases of the woman, cardiac group of 96 cases, 145 cases of pulmonary group. Adopted outside the peripheral venous blood $1 \mathrm{ml}$ by EDTA, the application of Bayer company ACS:180 immunoluminometric quantitative testing equipment, the use of the Bayer BNP box reagents and double-antibody sandwich immunofluorescence assay determination of plasma BNP concentration, the normal value of the $0-100 \mathrm{ng} / \mathrm{l}$. The application GEVivid5 systemic digital colour Doppler ultrasound conventional echocardiography, measured and recorded LVED and LVEF.

Results (1) The cardiac group, the average level of plasma BNP $(604.78 \pm 137.70) \mathrm{ng} / \mathrm{l}$, pulmonary group of BNP average (43.56 $\pm 32.73) \mathrm{ng} / \mathrm{l}$, significantly lower than the cardiac group $(p<0.01)$. Plasma BNP concentrations of heart failure severity increased can be drawn from the cardiac group, of which 28 cases of NYHA grade II, III grade 30 cases, 23 cases of grade IV, BNP levels (246.56 $\pm 883.42) \mathrm{ng} / \mathrm{l},(568.23 \pm 153.68) \mathrm{ng} / \mathrm{l}$, and $(1123.83 \pm 186.56) \mathrm{ng} / \mathrm{l}$. (2) Two groups of patients with plasma to comparison of LVEF, LVED and BNP levels, heart failure patients with BNP levels and LVED was positively correlated $(r=0.56, p<0.01)$, and LVEF was negatively correlated $(\mathrm{r}=-0.53, \mathrm{p}<0.01)$. 34 cases of NYHA grade II, 36 cases of gradeIII, 26 cases of grade IV, the LVED value, respectively $(56.4 \pm 5.8) \mathrm{mm}(61.7 \pm 6.5) \mathrm{mm}(65.4 \pm 3.7) \mathrm{mm}$. LVEF $(48 \pm 9) \%$, $(40 \pm 8) \%$, $(30 \pm 6) \%$.

Conclusions Compared with cardiac dyspnoea group and pulmonary breathing difficult group, BNP levels were significantly increased, and proportional to the severity of the BNP and heart failure, heart failure, BNP levels was a positive correlation was a negative correlation with LVEF and LVED, suggesting that the release of $\mathrm{BNP}$ directly related to excessive ventricular pressure overload, with the rise of ventricular pressure overload, ventricular myocytes increased secretion, and to determine the severity and changes in patients with heart failure by BNP. With the advent of the BNP rapid diagnostic tests, clinical detection of BNP levels has become more sophisticated, timely detection of clinical bedside quickly distinguish between dyspnoea, early diagnosis and rational treatment of patients with breathing difficulties to provide excellent technical support. 